\title{
Les recompositions de l'action publique en contexte transfrontalier
}

L'exemple du district hydrographique de l'Escaut

Helga-Jane Scarwell et Richard Laganier

\section{(2) OpenEdition}

\section{Journals}

Édition électronique

URL : http://journals.openedition.org/developpementdurable/1077

DOI : 10.4000/developpementdurable.1077

ISSN : 1772-9971

Éditeur

Association DD\&T

Référence électronique

Helga-Jane Scarwell et Richard Laganier, « Les recompositions de l'action publique en contexte transfrontalier », Développement durable et territoires [En ligne], Dossier 2 | 2003, mis en ligne le 01 novembre 2003, consulté le 02 mai 2019. URL : http://journals.openedition.org/ developpementdurable/1077 ; DOI : 10.4000/developpementdurable.1077

Ce document a été généré automatiquement le 2 mai 2019.

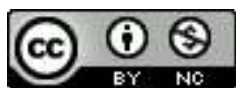

Développement Durable et Territoires est mis à disposition selon les termes de la licence Creative Commons Attribution - Pas d'Utilisation Commerciale 4.0 International. 


\title{
Les recompositions de l'action publique en contexte transfrontalier
}

\author{
L'exemple du district hydrographique de l'Escaut
}

Helga-Jane Scarwell et Richard Laganier

Les auteurs remercient le CNRS de son soutien dans le cadre du programme " Environnement, Vie et Sociétés ", projet intitulé "Gouvernance et territoires face à l'émergence des stratégies du développement durable : les espaces à forte contrainte environnementale de la Région Nord-Pas-deCalais ».

1 La notion de développement durable semble bénéficier depuis quelques années d'un contexte favorable à sa mise en œuvre tant au niveau local (diffusion de la notion et usage récurrent dans de nombreux discours politiques; intégration parmi les objectifs prioritaires des politiques territoriales, lois et réglementations, et surtout programmes incitatifs...), qu'aux niveaux communautaire et étatique.

2 L'observation et l'analyse d'un certain nombre de politiques locales élaborées dans un tel contexte posent toutefois la question de leur pertinence au regard des enjeux du développement durable et de leur capacité réelle à faire évoluer en profondeur l'action publique locale. En d'autres termes, elles soulèvent le problème de leurs impacts effectifs.

3 L'objectif de cette contribution est d'analyser comment dans un espace densément peuplé, le district international de l'Escaut, la question de la gestion transfrontalière de l'eau concerne le développement durable, en lui imposant des cadres territoriaux complexes et mouvants, et comment la mise en œuvre des principes de développement durable se construit sur l'émergence du souci de proximité et influence les politiques de l'eau.

4 En l'occurrence, la gestion de l'Escaut, fleuve transfrontalier, présente désormais un caractère multiforme, qui a pour conséquence de déplacer la scène du niveau du bassin versant vers celui du district hydrographique. 
5 À cet effet, on conçoit qu'une nouvelle organisation de l'espace et que de nouveaux modes d'action publique puissent servir de référence pour dépasser le cadre communal, considéré comme trop restreint pour gérer désormais une politique de protection durable de la ressource en eau au niveau communautaire et en concertation avec les acteurs intéressés.

6 La question qui se pose alors est celle de l'intégration des échelles spatiales et temporelles. Comment ces nouveaux espaces fonctionnels publics et/ou territoires émergents s'articulent-ils et interagissent-ils avec les territoires institutionnels existants? Ou éventuellement faut-il relativiser ces distorsions entre les territoires institutionnels politiques et l'hydrosystème (Lefèvre, 1995)?

7 Ces interrogations nous conduiront à envisager d'autres questions, à savoir si les habitudes de fragmentation du pouvoir qui s'inscrivaient dans une indépendance des territoires évoluent vers une coopération inter- et trans-institutionnelle, reconnaissant ainsi l'interdépendance des territoires et aboutissant finalement à une gouvernance de l'espace transfrontalier (Gaudin, 1996 ; Le Galès, 1995).

8 Enfin, l'État semble encadrer cette dynamique fonctionnelle en termes de coordination et de concertation, tout en produisant de nouvelles règles ou méthodes (Badie, 1995, Theys 2002). Celles-ci rendent-elles la frontière poreuse et affaiblissent-elles pour autant le rôle régulateur de l'État (Jouve, 1994 ; Levy, 1995 ; Raffestin, 1993)?

9 C'est à partir de l'intégration des échelles spatiales et temporelles et des dynamiques de proximité qui renvoient à la mise en œuvre a priori ou ex-post de nouveaux modes de gouvernement que l'on pourra alors formuler l'hypothèse selon laquelle les principes du développement durable, à travers des pratiques judicieuses, offrent aux pouvoirs publics locaux une opportunité pour redonner à l'environnement ses multiples dimensions spatiales et sociales.

\section{D'une gestion sectorielle à une gestion globale durable : les grandes lignes de la Directive-cadre sur l'eau (DCE)'}

10 Jusqu'au milieu des années soixante ${ }^{2}$, la gestion de l'eau a été conçue de façon sectorielle (Loriferme, 1987 ; Valiron, 1990) en s'inscrivant dans des logiques économiques locales, régionales, voire nationales, peu soucieuses de la dimension environnementale et de la pérennité de la ressource en eau.

11 Depuis 1975, l'Union Européenne a élaboré plus d'une trentaine de directives ou décisions relatives à l'eau, traduisant l'évolution d'une approche sectorielle vers une approche intégrée de ses modalités de gestion. La prise en compte de ces directives par le droit français traduisant une vision plus transversale (environnement, économie, social) et plus territorialisée, est décrite dans la loi sur l'eau du 3 janvier $1992^{3}$. En effet, celle-ci reconnait une nouvelle circonscription de gestion, le bassin versant, qui se substitue, en la matière, aux découpages administratifs traditionnels. Désormais, à l'intérieur de cette entité, une planification et une gestion concertée et intégrée de l'eau remplacent le cadre traditionnel de la gestion sectorielle, grâce aux outils que sont le SDAGE ${ }^{4}$, pour les six bassins hydrographiques, et les SAGE ${ }^{5}$, pour les sous-bassins versants, dont l'objet est une gestion intégrée de la ressource en eau. 

général pour une politique de l'eau qui intègre aussi bien ses usages que ses effets perturbateurs ou destructeurs, il n'en demeure pas moins que le bassin versant transfrontalier n'était pas pris complètement en compte et concernait plusieurs territoires. Ce caractère pluriel impliquait des zones de chevauchement et/ou des carences dans l'intervention. Or l'importance des enjeux impose une gestion cohérente de l'ensemble du bassin hydrographique.

Par ailleurs, les directives communautaires et les décisions relatives à l'eau étaient adoptées selon une double approche, qui consistait d'une part, à lutter contre les rejets de substances dangereuses dans l'environnement et, d'autre part, à définir des normes de qualité concernant des zones particulières.

C'est pourquoi il importait d'harmoniser l'ensemble des textes au travers d'une directive cadre.

La directive établissant un cadre pour une politique communautaire dans le domaine de l'eau (DCE) a été adoptée sous la présidence française le 23 octobre 2000 et publiée le 22 décembre 2000. Elle vise à établir un cadre global pour la protection des eaux continentales, souterraines et côtières : le district hydrographique.

Elle conforte le dispositif français qui organise la gestion de l'eau par grand bassin hydrographique, avec des comités de bassin qui rassemblent les représentants des collectivités territoriales, des usagers et des associations, ainsi que des services de l'État. Si elle s'inspire largement du système français, elle en diffère sensiblement par plusieurs aspects, et notamment l'obligation de résultat, sous peine de contentieux, la prise en compte de la biologie dans la qualification de l'état des eaux, et la participation forte du public à tous les stades de la procédure.

Figure 1. Vers une approche intégrée de la gestion de l'eau

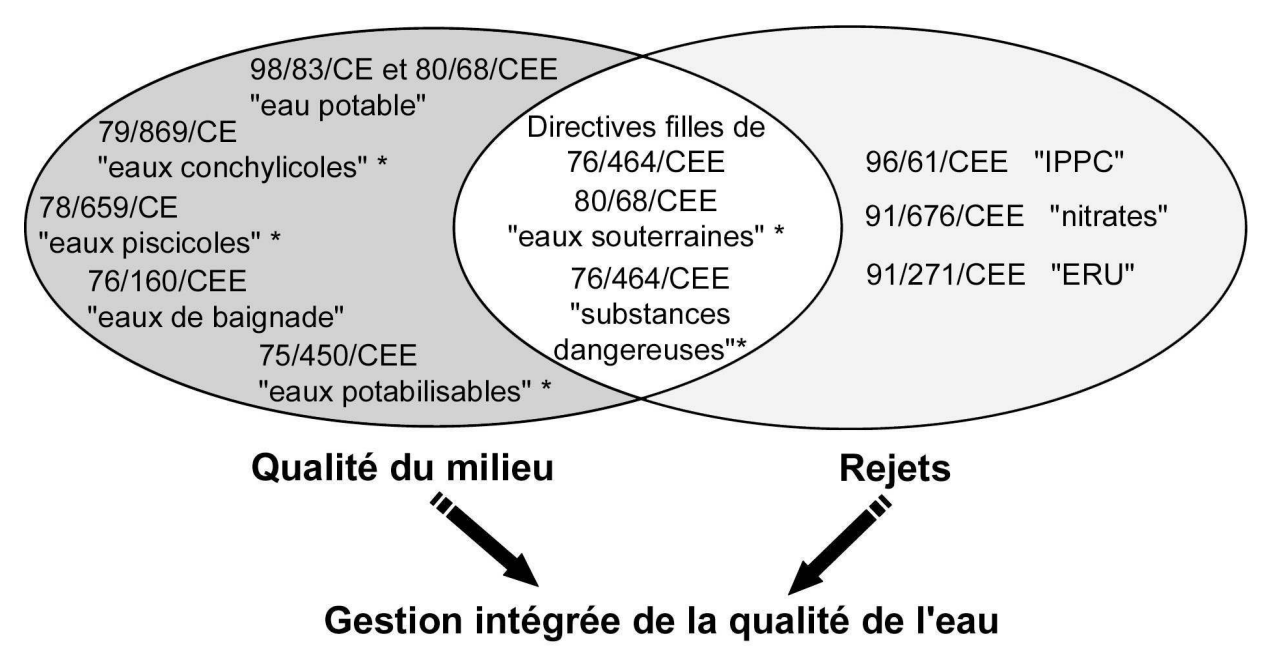

* Directives destinées à être intégrées dans la directive cadre 2000

(d'après Barbara Bour-Desprez, 2003)

La directive propose une approche intégratrice en prévoyant de décrire directement les milieux aquatiques à partir des habitats et des espèces qui les composent. L'objectif à atteindre devient «le bon état écologique» qui doit être compatible avec une pression humaine raisonnable. 
- une approche économique qui se traduit par une analyse de l'utilisation de l'eau devant servir d'aide à la décision pour l'optimisation des choix de mesures, afin de justifier de la dérogation aux objectifs fixés par la Directive et pour l'évaluation du niveau de recouvrement des coûts des services de l'eau. Le rôle de l'analyse économique doit être intégré tout au long du processus de mise en place de la DCE ;

- la participation (art. 14 de la DCE), qu'il faut entendre comme la participation active ${ }^{6}$ des parties intéressées, la mise à disposition des informations et la consultation du public. Précisons que la participation active du public à la mise en œuvre de la politique de l'eau est fortement encouragée. Elle se situe dans le droit fil de la convention d'Aarhus ${ }^{7}$.

Figure 2. Modalités de la participation

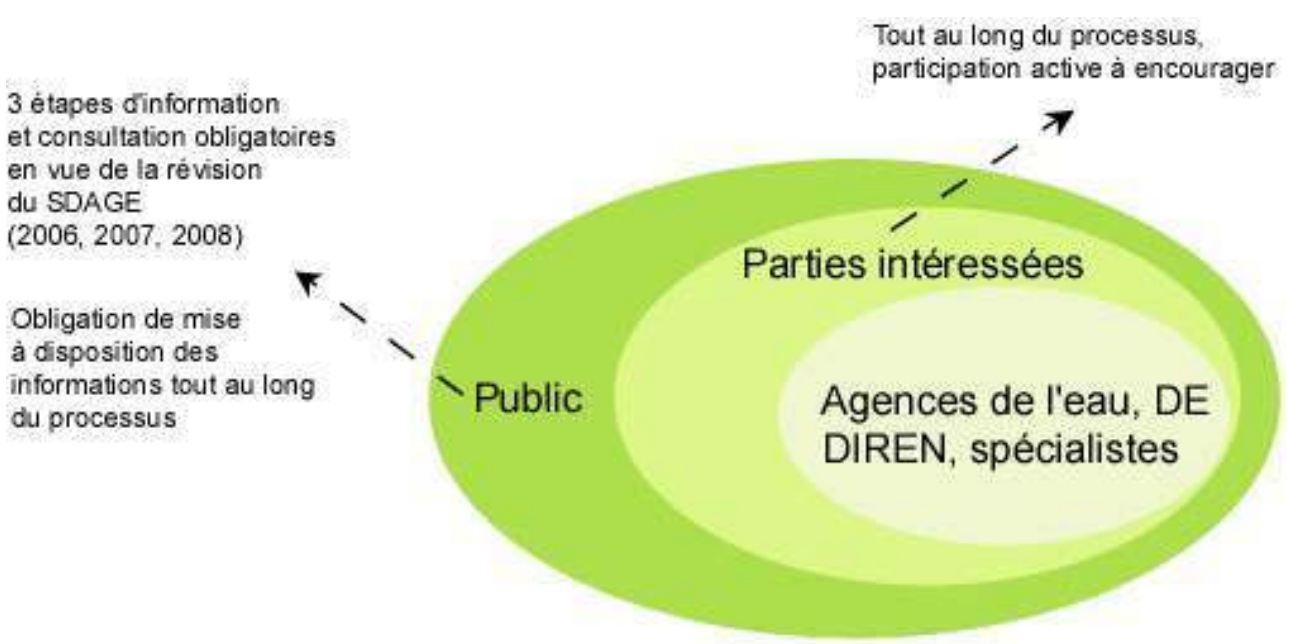

D'àprés Coralie Noél, Ingénieur chargée de mission - Direction de l'eau

Source : Noël, 2003

En conclusion, il s'avère que cette directive, en introduisant autant de notions nouvelles, doit encore être accompagnée d'un lourd travail au niveau européen de façon à en préciser les concepts. C'est pourquoi, le Gouvernement français a décidé de lancer un " grand débat », afin de déterminer en concertation avec l'ensemble des acteurs de l'eau les aménagements qui devraient être apportés à la politique nationale pour faire face à ces enjeux. Ce débat devrait prendre en compte les réflexions qui pourront résulter des chantiers en cours sur la décentralisation, la charte de l'environnement ou la mise au point de la stratégie nationale du développement durable. Ceci étant, la prise en compte de la durabilité de la ressource en eau est non seulement réaffirmée mais renforcée par la prise en compte "du bon état écologique de la ressource », par la clarification des compétences et des responsabilités de chacun afin de maintenir une politique décentralisée, par l'information et la participation du public à la politique de l'eau, l'amélioration de la représentativité des différents acteurs au sein des institutions existantes, enfin la planification et la cohérence de la politique de l'eau avec les autres 
politiques publiques. Il faudrait bien évidement s'interroger notamment sur la notion de "bon état écologique ", ou plus globalement sur la pertinence de la DCE et de sa mise en application par la France par rapport aux objectifs du développement durable. Rappelons que nous nous attacherons plus particulièrement à la gouvernance et à ses moyens.

Parce que la mise en œuvre de la DCE sur l'Escaut, district hydrographique transfrontalier, pose la question de l'intégration des échelles spatiales et temporelles et plus globalement de l'organisation des territoires et des réseaux d'acteurs et de leurs impacts spatiaux, nous l'aborderons sous l'angle de l'articulation et des conflits de logiques d'acteurs. Notre objectif est d'analyser les interactions de ces nouveaux espaces publics et territoires émergents, avec les territoires existants.

\section{2. Échelles et articulations}

L'Escaut, qui s'écoule sur près de $350 \mathrm{~km}$ du Nord de la France aux Pays-Bas correspond à l'un des bassins versants les plus importants d'Europe $\left(21683 \mathrm{~km}^{2}\right)$. Cependant, son débit est relativement faible, environ $80 \mathrm{~m}^{3} . \mathrm{s}^{-1}$ à Anvers (i.e. moins de $51 . \mathrm{s}^{-1} \cdot \mathrm{km}^{2}$ ) comparé au Danube $\left(6500 \mathrm{~m}^{3} \cdot \mathrm{s}^{-1}\right)$, au Rhin $\left(2200 \mathrm{~m}^{3} \cdot \mathrm{s}^{-1}\right)$ et à la Meuse $\left(330 \mathrm{~m}^{3} \cdot \mathrm{s}^{-1}\right)$. Son bassin se répartit entre la France ( $31 \%$ du total), la Wallonie (17\%), la Flandres ( $43 \%)$, Bruxelles $(1 \%)$ et les Pays-Bas ( $8 \%)$. Sa population, qui dépasse les 10 millions d'habitants, est très irrégulièrement répartie, la densité ne dépassant pas les 10 habitants par $\mathrm{km}^{2}$ sur la Haute Lys ou en Zélande et excédant les $2000 \mathrm{hab} . / \mathrm{km}^{2}$ à Anvers, Bruxelles ou Lille. Après plusieurs siècles d'un important développement économique, peu concerné par les impacts à la fois qualitatifs et quantitatifs sur l'hydrosystème qu'il pouvait engendrer, a émergé depuis plusieurs décennies une prise de conscience des enjeux hydro-écologiques et plus récemment, la nécessité de se coordonner pour mettre en œuvre un plan de gestion à l'échelle internationale. Ces enjeux concernent à la fois :

- la pollution des eaux par les villes, les industries et l'agriculture intensive;

- le risque d'inondation dans un contexte d'étalement urbain en zone inondable, d'imperméabilisation des sols et de conquête historique des zones humides ;

l'épuisement de la ressource en eau face à une demande croissante et à des déséquilibres régionaux en matière de disponibilité en eau. 


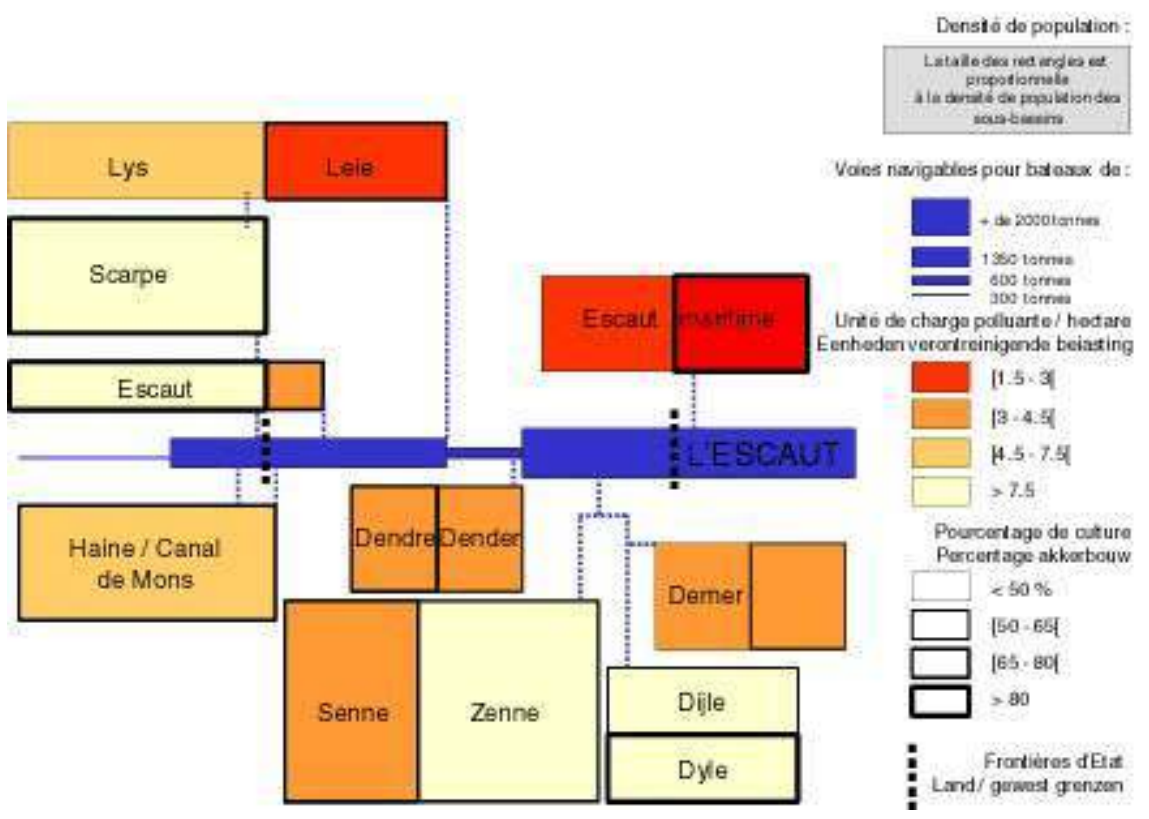

Source : Laganier, 2003

La figure 3 met en évidence cette disparité des enjeux à l'échelle du bassin versant et souligne ainsi la nécessité de réfléchir à une meilleure articulation des échelles pour assurer une gestion intégrée de l'eau. C'est ce que propose notamment la démarche entreprise à travers la Commission Internationale de l'Escaut (CIE), à l'échelle d'un district hydrographique qui dépasse les frontières du bassin versant « naturel ». Les cours d'eau côtiers français et flamands sont ainsi intégrés dans le dispositif.

Figure 4. Le district de l'Escaut

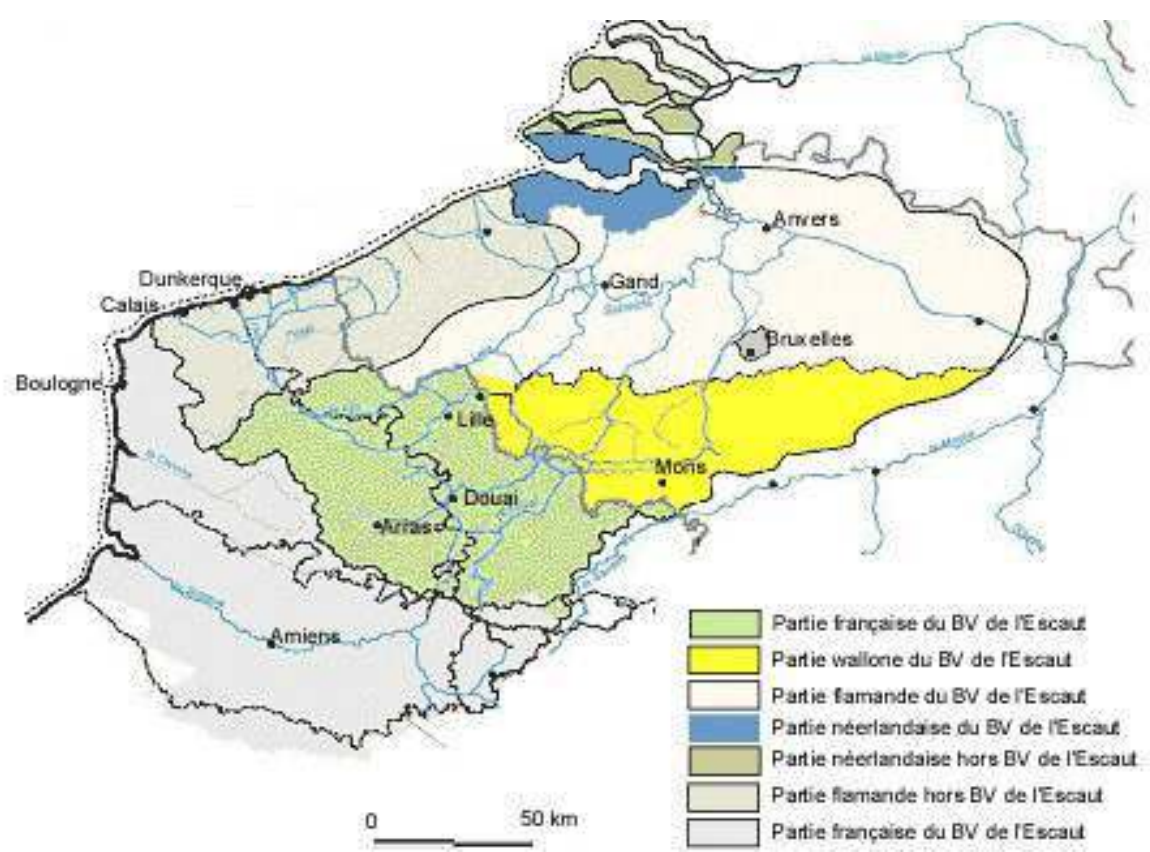

Source : CIE, 2003 
L'exemple transfrontalier du district international de l'Escaut constitue une modalité significative de mise en adéquation de l'hydrosystème avec les territoires institutionnels. Si la question de la distorsion entre les territoires concernés et le territoire fonctionnel semble résolue dans la mesure où, désormais, le district hydrographique devient le niveau pertinent de gestion et de protection de la ressource en eau, il convient de mettre en évidence la façon dont les échelles s'articulent en vue de parvenir aux objectifs fixés par la DCE.

25 Par ailleurs, cette mise en cohérence de l'échelle des problèmes avec celle de la décision pourrait tendre à une redéfinition du rôle et de la place de l'État dans la production des politiques. Mais aussi, l'intégration d'échelles plus nombreuses complexifie le jeu des acteurs. En effet, l'émergence de ce nouvel espace se double de l'avènement de nouveaux partenaires susceptibles d'induire des problèmes de régulation inédits auxquels la gouvernance territoriale pourrait répondre par un réaménagement de la gestion de l'action publique.

Cinq échelles territoriales sont concernées par la mise en œuvre de la DCE sur le district hydrographique de l'Escaut, partie française (figure 5).

- L'échelle européenne où a été élaborée la DCE ;

- L'échelle nationale où s'applique la DCE, sachant que seuls les États sont responsables de cette mise en œuvre dans les délais impartis. Ils doivent en fixer l'encadrement juridique. ;

- L'échelle transfrontalière illustrée par la CIE. Les États concernés par l'Escaut ont inscrit la coopération transfrontalière dans l'accord de Gand créant la CIE. Elle est chargée d'élaborer le plan de gestion du district, qui énonce des avis et des recommandations ${ }^{8}$ en coopération avec les États concernés ;

- L'échelle régionale du Comité de Bassin Artois-Picardie (CB) qui élabore le SDAGE en conformité avec le futur plan de gestion, tout en couvrant un domaine plus large que le plan de gestion (exemples de la prévention des risques d'inondations, de l'extraction de granulats, de la sécurité de l'alimentation en eau potable, ...) ;

- Enfin, l'échelle locale de la Commission locale de l'eau (CLE). 
Figure 5. Échelles et articulations des scènes de concertation et de décision dans le domaine de l'eau - District de l'Escaut

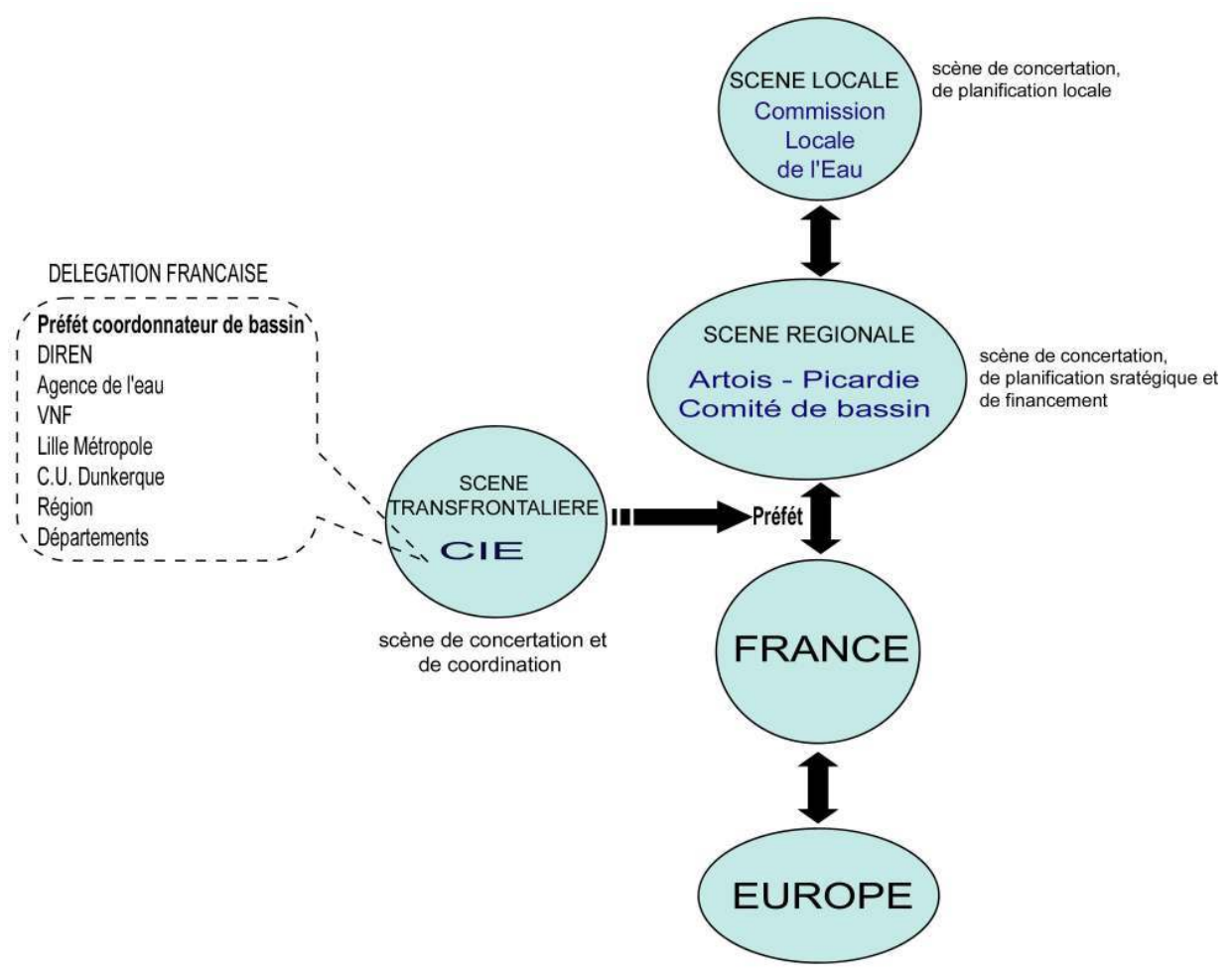

Source : Scarwell/Laganier 2003

\subsection{Comment s'organisent les modalités de coopération entre ces échelles et comment doit-on qualifier cette dynamique?}

En pratique, il ne s'agit pas pour chaque niveau d'agir en toute liberté mais au contraire de se conformer à un certain nombre de principes directeurs communs définis tant au niveau communautaire qu'au niveau national.

Pour l'application de la DCE, l'État français a signé à Gand le 3 décembre 2002 un nouvel accord international sur l'Escaut en vue de «» renforcer la coopération existante entre les États et Régions concernés par la protection et l'utilisation des eaux du district hydrographique international de l'Escaut $»^{10}$.

\subsection{La Commission internationale de l'Escaut (C.I.E)}

La mise en œuvre de la DCE qui établit un cadre pour une politique communautaire dans le domaine de l'eau nécessite, au sein du district hydrographique international de l'Escaut, selon les domaines géographiques et les thèmes à traiter, une coordination multilatérale, bilatérale ou nationale ${ }^{11}$. Cette coordination s'effectuera dans le cadre de la CIE (Commission internationale de l'Escaut).

Cette organisation inter-gouvernementale doit être porteuse d'une dynamique capable de produire du consensus dans l'identification des prescriptions communes en termes de données, de rapports et de produits qui passera par une harmonisation avec les divers 
partenaires transfrontaliers pour contribuer à la transparence et à la coordination de la gestion de l'eau au niveau du district international.

Ceci étant, la diversité des acteurs ne peut à elle seule garantir son succès ni d'ailleurs celui des procédures nouvelles qui les sous-tendent (Leca, 1996). Il ressort que plus l'étendue du territoire considéré sera importante, plus il sera nécessaire d'assurer des liens de cohésion entre ses composantes.

En effet, le glissement progressif d'un système de relations associant dans un cadre national français l'État et les collectivités territoriales vers un autre système de relations concernant l'Europe, les États, les collectivités territoriales, les intercommunalités et des acteurs privés, incite à préciser les interactions entre ces «niveaux territoriaux ", mais surtout entraîne une modification du jeu des relations pour se rapprocher d'un objectif d'optimisation de la satisfaction qui suppose une connaissance et une articulation des territoires concernés et enfin, une évaluation des résultats.

$33 \mathrm{La} \mathrm{CIE}^{12}$ réunit la Région flamande, la Région wallonne, la Région de Bruxelles capitale, l'État fédéral Belge, le Royaume des Pays-Bas et la France. Ceux-ci menaient déjà des actions de coordination autour de la qualité des eaux et, plus particulièrement, sur un système d'alerte des pollutions accidentelles et sur un réseau de mesures homogènes. Dorénavant, cette coordination doit renforcer l'articulation des échelles transfrontalières et nationales et favoriser la concertation avec l'ensemble des acteurs publics et privés concernés.

Chaque État concerné par l'Escaut est représenté au sein de la CIE par une délégation dont la composition varie en fonction des parties contractantes. Par ailleurs, la structure fédérale de certains États concernés augmente le nombre de délégations présentes.

En ce qui concerne la France, la délégation française est désormais menée par le préfet coordonnateur de Bassin, qui doit veiller à l'harmonisation des calendriers des travaux entre la partie nationale et l'ensemble du district international et en proposer les adaptations qu'il jugera utiles. Elle est composée des principaux services de l'État ${ }^{13}$ intervenant dans la gestion intégrée de l'eau, auxquels il faut ajouter des représentants des collectivités locales ${ }^{14}$ concernées. Également, la CIE reconnaît la qualité d'observateur à certaines organisations, dont les ONG, pour autant qu'elles aient des points communs avec la mission de la CIE. À ce titre, elles peuvent participer aux réunions de la Commission sans pour autant disposer d'un droit de vote.

La délégation française au sein de la CIE participe avec les autres délégations étrangères à l'élaboration des avis et recommandations qui, pour le cas de la France, seront transmis ensuite au Comité de Bassin, lequel les prendra en compte pour la révision du SDAGE.

Enfin, la CLE répercutera à son tour les principales orientations définies par le SDAGE pour l'élaboration du SAGE.

La volonté de l'Union européenne et des États de créer des réseaux de coopération transfrontalière semble s'inscrire dans une perspective de renforcement des stratégies de coopération que les seuls référentiels communs ne sauraient imposer. En effet, si cet espace public de coopération entre acteurs publics/privés nationaux et étrangers est mis en place au niveau de l'échelle transfrontalière en raison de l'interdépendance des autres échelles, il ne s'agit pas de sous-estimer les résistances liées aux barrières juridiques et socioculturelles de la frontière. La CIE doit concourir à l'élaboration d'un seul plan de gestion ${ }^{15}$ pour l'ensemble du district hydrographique de l'Escaut, mais ne dispose pour ce faire que du pouvoir d'émettre des avis et recommandations. Il ne s'agit en fait que d'une 
scène, d'une instance de concertation et de coordination. Chaque partie contractante répercute sur son territoire les dispositions qui le concernent. De même, le principe de la solidarité de l'ensemble des acteurs du district hydrographique de l'Escaut ne s'applique pas. En n'émettant que des avis et recommandations, elle ne dispose pas du pouvoir de décider d'une solidarité financière entre tous les acteurs du district. Elle ne bénéficie que d'un budget limité et la plupart des opérations sont financées par le biais de programmes européens Interreg 3. Sa marge de manœuvre est donc limitée.

On aurait pu imaginer que la réalisation d'un seul plan de gestion sur l'ensemble du district hydrographique s'accompagne d'un réel pouvoir de décision de la CIE, illustrant ainsi une coopération transfrontalière plus poussée, et d'une gestion à ce même niveau. En définitive, si l'émergence d'espaces publics transfrontaliers est propice à l'expression singulière de rapports de forces inédits et structurés par l'histoire des lieux et des acteurs, il n'en sera rien dans notre cas en raison des prérogatives de la CIE qui se focalisent sur des avis et des recommandations. La portée de ces rapports de force pourraient être relativement limitée.

\section{Le district international de l'Escaut, espace de gouvernance territoriale?}

40 L'Union européenne s'est engagée dans une démarche ${ }^{16}$ visant à faire évoluer les processus de décision dans l'Union en cassant l'idée selon laquelle "seuls la Commission européenne et l'État pensent, et les autorités régionales et locales exécutent ${ }^{17}$. Dans cette perspective, elle incite les États à développer la coopération pour favoriser l'articulation des différents acteurs et pour installer une gouvernance à plusieurs niveaux, ainsi que dans le sens d'une meilleure intégration des actions ayant un impact sur le territoire européen et pour lesquelles les autorités régionales et locales sont presque toujours concernées.

41 Ainsi, les nouveaux mécanismes de coopération entre les différents niveaux de décision devraient reposer sur une logique de compétences partagées et non hiérarchisées.

Le mot « gouvernance » est actuellement à la mode et nous ne prétendons ni le redéfinir à notre tour ni en refaire l'historique, mais il importe de nous référer à une conception et à des repères pour mieux en mesurer les implications.

La conception européenne de la gouvernance à laquelle nous faisons ici référence est celle définie par le Livre blanc sur la gouvernance européenne. Comme le souligne Beate Kohler-Koch et Fabrice Larat, la " gouvernance communautaire n'est pas seulement déterminée par la structure de la communauté et par ses particularités, mais elle est aussi influencée par la perception des principes régissant un ordre politique légitime qu'ont les acteurs participant à cette gouvernance (...) Dans son essence, la gouvernance concerne la matière et les moyens par lesquels les préférences en partie divergentes des citoyens se trouvent traduites dans les choix et mesures de nature politique, de telle façon que la pluralité des intérêts présents au sein de la société soit transformée en action unitaire et que les différents acteurs sociaux y consentent et s'y retrouvent ». L'usage de ce mot renvoie tant aux principes de bonne gouvernance qu'aux exigence élémentaires de la démocratie, à savoir l'accès à l'information, le fait que les gouvernants doivent favoriser la transparence des décisions en rendant des comptes aux citoyens, et qu'enfin ces derniers aient prise sur les décisions qui les concernent. 

leur pratique effective. Dès lors, comme le souligne Pierre Calame, "le pouvoir de proposition et l'organisation du cycle d'élaboration, de mise en cuvre et d'évaluation des politiques publiques sont au cour de la gouvernance » (Calame, 2003).

\section{l'analyse des conditions de la participation et sur l'appropriation et/ou la ré-} appropriation par la société civile et les élus locaux des pratiques mises en œuvre pour assurer un développement durable de la ressource en eau. Dans cet esprit, il nous apparaît que la question de la régulation des problèmes liés à l'eau au sein du district international de l'Escaut est propice à une analyse en termes de "proximité ", d'autant que cette régulation s'inscrit dans des controverses de natures variées: diversité des modes de perception des problèmes, de la nature des intérêts concernés, du degré de réversibilité ou d'irréversibilité des phénomènes et du degré de stabilisation de la connaissance scientifique des problèmes (Godard, 1994). L'enjeu est alors une mobilisation plus large des acteurs, la construction de nouveaux lieux de rencontre et d'organisation des politiques (Assouline et Blancher, 2003), une meilleure articulation entre recherche et décision, afin de mieux valoriser le capital cognitif et/ou le recours à des stratégies innovantes.

Comment, par des systèmes de médiation, l'État essaie-t-il de répondre aux aspirations qualitatives des citoyens? Comment gérer la proximité sans détourner l'attention de questions non formulées mais portant des enjeux forts pour l'avenir? Quels sont les apports de cette dynamique européenne, voire internationale, pour les citoyens? Quels sont les arguments utilisés pour démontrer un double dividende? Enfin, quelles sont les actions de sensibilisation sur lesquelles l'État et les collectivités s'appuient pour responsabiliser les populations?

que les décisions doivent être prises au niveau le plus proche du citoyen (principe de subsidiarité18), elle ne prévoit aucun moyen pour mettre en œuvre ce principe, ce qui ne facilite pas la transparence. Ces objectifs ne sont donc pas à l'abri d'ambiguïtés liées au cadre juridique et institutionnel proprement dit.

Ce principe de subsidiarité permet certes de limiter les excès de la centralisation, tout en évitant les chevauchements de compétences, mais il n'empêche pas la tendance des institutions à exercer leurs attributions selon le principe d'exclusivité, «mal adapté à la complexité de l'action publique» (Freyss, 2003). Enfin, ce principe ne devrait pas seulement s'appliquer aux rapports entre les collectivités publiques plus larges et les collectivités publiques plus petites, mais aussi aux rapports entre les autorités publiques et la société civile. Il appartient donc aux États de favoriser la mise en place de cette gouvernance par le biais de l'information et de la participation du public.

lors, le débat sur la gouvernance à plusieurs niveaux devient difficile et biaisé dans la mesure où ceux qui y participent choisissent souvent l'interprétation de la subsidiarité qui les avantage, ce qui conduit parfois à des malentendus. En prolongeant notre réflexion, nous pourrions nous demander s'il appartient à l'instance supérieure, au regard du respect de l'autonomie de l'instance inférieure, d'intervenir si cette dernière ne remplit pas suffisamment sa mission (de la Malène, 1997) ? C'est pourquoi certains auteurs préfèrent parler de "subsidiarité active ${ }^{19}$ ». Celle-ci s'intéresse davantage aux « objectifs, aux critères, et aux dispositifs de travail », alors que la subsidiarité, sans autre 
qualificatif conduit «à lister et à délimiter des compétences déléguées au niveau du dessus » (Calame, 2003, p. 188).

D'entrée de jeu, la subsidiarité active met l'accent sur la manière d'élaborer de façon partenariale des solution pertinentes dans la gestion des problèmes et satisfaisant aux obligations de résultats. Toutefois, pour la mise en œuvre de la DCE, l'Union européenne a tenté de déterminer des principes simples et clairs et des méthodes concrètes pour progresser à partir d'une démarche fondée sur la coopération. En effet, les États membres doivent encourager « la participation active de toutes les parties concernées par la mise en œuvre de la présente directive, notamment à la production, à la révision et à la mise à jour des plans de gestion de district hydrographique ». Il n'en demeure pas moins que la gouvernance n'est pas une donnée, mais se construit à travers les interactions des acteurs et doit donc être entretenue en permanence. Comme le souligne Mireille Pongy, «la gouvernance renvoie (...) à une acceptation relationnelle du pouvoir, fondée sur la notion d'échange, et non à une vision substantialiste du pouvoir comme propriété ou comme lieu» (Pongy, 1997, p. 112).

51 En ce sens la légitimité de la gouvernance dépend de son enracinement culturel; il appartiendra donc à chaque État de dire comment il s'organisera pour atteindre les buts qu'il se sera fixés et comment sera organisé le partenariat entre les acteurs.

52 Finalement, le fondement de la gouvernance européenne n'est-il pas celui des autres niveaux? Mais s'agit-il là véritablement d'une question pertinente, puisque les deux cas de figure cohabitent et qu'en outre, un grand nombre d'innovations institutionnelles relève comme le souligne Pierre Jacquet, «d'une zone grise » entre les deux (Jacquet, 2000, p. 288) ? Là encore, l'analyse de cette dynamique croisée illustrera les changements institutionnels qui sont à la fois le signe de l'adaptation à certaines innovations et le moteur d'autres innovations.

\section{De la complexité de mener une politique transversale et partenariale au niveau transfrontalier}

53 Cette analyse n'en est qu'à un stade exploratoire en raison de son déroulement en temps réel. En effet, elle ne constitue qu'une première marche d'un édifice d'ensemble qui articule, au plan de la gouvernance, les différents niveaux d'échange. Il est donc encore trop tôt pour évaluer l'impact réel du dispositif mis en place, mais il est possible dès à présent d'analyser les articulations entre les différentes échelles et les interactions entre les acteurs.

54 Il est bien évident que certaines questions resteront suspendues à une analyse fine dans le temps qui évaluerait l'ensemble du processus de coordination.

L'État et l'espace national ont été souvent les cadres privilégiés des régulations publiques, politiques et sociales. L'interdépendance des politiques au niveau communautaire, voire mondial, a favorisé la diversité des dynamiques sociales et développé d'autres modes d'action publique. Les systèmes évoluent donc pour s'adapter à de nouvelles exigences et représentations, mais comme le souligne $\mathrm{P}$. Calame, «la gouvernance est par nature et par vocation un système d'évolution lente» (Calame, 2003, p. 18). C'est pourquoi il convient de mettre en évidence, d'une part, les évolutions de ce système, en soulignant les retournements ou les ré-ajustements des principes d'action publique préexistants autour 
des principes nouveaux pour en donner une lisibilité et d'autre part, de vérifier que l'État met en conformité son action avec les valeurs qu'il énonce.

Le transfrontalier met en scène de multiples structures de gouvernement qui pourraient favoriser des approches plus transversales, engendrant à leur tour un processus d'apprentissage de l'action publique où les interventions proviendraient de lieux et de niveaux de la décision politique à la fois fragmentés, imbriqués et contractualisés.

Ainsi, l'architecture de la coopération transfrontalière peut apparaître complexe, parce qu'elle aligne plusieurs niveaux territoriaux. L'ambiguïté réside dans le fait que ces questions de coopération transfrontalières, plutôt « locales », sont surtout traitées par les administrations déconcentrées de l'État, même si des collectivités territoriales y siègent. La présence au sein de la délégation française de la CIE des Communautés urbaines de Lille et de Dunkerques, ne traduit pas nécessairement un juste partage des tâches, mais renforce une stratégie commune au regard de certains enjeux (transferts d'eau, inondation ...).

Par ailleurs, les différences entre les systèmes politiques fédéraux et unitaires accentuent la place mineure dévolue aux collectivités françaises. Finalement, le dispositif mis en place engendre un enchevêtrement institutionnel lié à la diversité des stratégies et des enjeux des différentes parties.

$\mathrm{Au}$ demeurant, il faut encore souligner un autre point lié à la démocratie de proximité dont l'Europe et l'État français se sont faits l'écho. Si la prise en charge des affaires locales par les collectivités territoriales semblait justifiée par la "proximité », l'encadrement juridique de la coopération transfrontalière demeure coordonné par l'État. Les préoccupations de démocratie, comme le soulignent Guy Saez et Michel Bassand «ne semblent ni l'objet ni le cadre de référence explicite des formes actuelles de la gouvernance » (Saez et Bassand, 1997, p. 8). Quand bien même les collectivités invoqueraient le déficit démocratique dont souffrent parfois les institutions de coopération transfrontalière, la nécessité de créer des espaces de solidarité ou un développement concerté, l'encadrement juridique de la coopération veille à ne pas favoriser «l'affaiblissement de l'hégémonie de la normativité étatique ", déjà concurrencée par la construction communautaire (Robert, 1995), malgré l'émergence d'autres formes de coopération de proximité (COPIT, $\left.\mathrm{TSC}^{20}\right)$ à des échelles plus grandes.

60 En réalité, la régulation croisée demeure partielle en raison de la tutelle exercée par le préfet qui reste parfois nécessaire et qui favorise une forte implication de ses services dans la gestion du projet à tous les niveaux. Face à cette imbrication des échelles, le Préfet constitue en quelque sorte l'élément permanent qui garantit la cohésion tant verticale qu'horizontale (Theys, 2002).

61 La simple présence d'ONG ou de collèges d'usagers à chaque échelle ne saurait consacrer à elle seule l'existence d'un réel partenariat qui implique des possibilités d'initiatives de la part de chaque partenaire et qui ne peut se réduire à une invitation à participer.

62 Toutefois, est-ce à dire qu'il n'existe pas de concurrence des autres acteurs ? Là encore, le nombre et la force des structures de l'État tendent à lui conserver une place prééminente. Rappelons qu'au sein de la CIE, ce sont les États qui fixent eux-mêmes les modalités du dialogue. Nous analyserons ultérieurement la nature de ce partenariat en tentant de définir la marge réelle de manœuvre des acteurs et la délimitation des sphères publique et privée, et enfin, la part d'initiative de chaque partenaire. 

en cohérence des échelles où les problèmes se posent, la complexité apparente de ce nouveau cadre de référence n'est que très relative, en raison de la présence à chaque niveau des services de l'État et de représentants de l'Agence de l'eau. Ainsi, quelle que soit la configuration, l'État, par le biais du préfet et de ses services, maintient une cohérence tant horizontale que verticale. La DIREN assure la coordination internationale, l'Agence de l'eau la coordination avec le niveau national, enfin le préfet est chargé de la coordination générale. Ce qui laisserait penser que l'État, par le biais du préfet, se cantonne à ses missions régaliennes et ajuste les différentes temporalités de l'action publique. En ce sens, la DCE permet de reposer la question de la place et du rôle de l'État en relation avec les nouveaux espaces de coopération et de décision qui s'élargissent et se complexifient.

À ce stade de l'étude, le contexte transfrontalier ne met pas en évidence certaines tendances spécifiques dans la fabrication des politiques publiques que les contextes nationaux - pris séparément - révèleraient moins clairement.

\subsection{Les modalités de la participation en France pour la mise en œuvre de la DCE ou les réponses institutionnelles au défi de la gouvernance}

Si la DCE énonce dans son préambule que «le succès de la directive (...) requiert l'information, la consultation et la participation du public, y compris des utilisateurs ", la réalité est plus nuancée, car comme nous l'avons déjà souligné, elle renvoie à la subsidiarité nationale pour les moyens mis en œuvre en amont, en vue d'une consultation effective du public à partir de 2006. Ainsi, il appartient aux États d'organiser le calendrier de ces consultations et de définir les modalités de la participation du public. De façon précise, la DCE demande qu'après la réalisation de l'état des lieux, les États procèdent à trois consultations du public : la première avant la fin de 2006 sur le calendrier des travaux d'élaboration du plan de gestion, la seconde avant fin 2007 sur l'identification des problèmes principaux et la troisième fin 2008 à propos du projet de plan de gestion.

Que recouvre le terme « public »?

67 Par ce terme, l'Union européenne fait référence à celle, très large, de la convention d'Aarhus, ce qui va au-delà des consultations précédemment faites lors de l'élaboration du SDAGE.

S'ils souhaitent satisfaire à l'objectif de transparence ${ }^{21}$ et d'appropriation des stratégies, il est préférable que les États organisent une information sur la gestion de l'eau suffisamment synthétique et compréhensible par des non initiés ${ }^{22}$. A cet effet, avant l'adoption de l'état des lieux par les comités de bassin concernés, une consultation du public devrait être réalisée courant 2004, afin de recueillir les observations éventuelles sur ces documents. Parallèlement, une information du public devrait en France être préparée en s'appuyant notamment sur les relais locaux que constituent les collectivités territoriales, les organismes consulaires et les associations.

Le gouvernement français a segmenté le débat pour la mise en œuvre de l'article 14 de la DCE en trois phases ${ }^{23}$ :

- Une première phase nationale, quasiment achevée, a permis des contacts bilatéraux avec les représentants nationaux des principaux acteurs, afin de préciser les enjeux et le 
champ du débat local. Des contributions écrites ont été fournies ;

- Une deuxième phase de débat local, dans le courant du deuxième trimestre 2003, est placée sous l'égide des comités de bassin, ainsi qu'autour de l'initiative de régions ou de départements, voire de groupements de ces collectivités qui partageraient des préoccupations voisines. Un document de référence a été établi sur la base des enseignements inspirés par la première phase ;

- Une troisième phase associera à l'automne le "grand public» au travers d'un questionnaire largement diffusé et d'initiatives décentralisées. Cette phase est en cours de mise au point avec l'appui de la Commission Nationale du Débat Public.

La synthèse sera faite à la fin de l'année en liaison avec le Parlement lors de rencontres nationales dont l'objectif sera de proposer des recommandations qui serviront de base à la construction d'une politique de l'eau rénovée et d'un plan d'action.

71 Ce faisant, dans l'hypothèse où chacun pourrait avoir une compréhension différentes des termes de "public » et de " participation", toute réflexion à ce sujet ne saurait faire l'économie d'un questionnement sur trois points : qui sont les acteurs de ce débat et de ces concertations et à quel moment doivent-ils participer et avec quelle régularité ?

À ces trois questions, s'en ajoute une quatrième relative à la gouvernance.

En effet, ces règles du jeu renouvellent-elles le paradigme centre-périphérie ${ }^{24}$, induisant par cela même une restructuration de l'action publique? Plus globalement, l'État perd-il de sa centralité en ouvrant des possibilités de structurations horizontales, éventuellement verticales à des niveaux intermédiaires?

73 De plus, si l'on peut imaginer des modes de gouvernance ${ }^{25}$ territorialisés, quels sont les mécanismes et les processus en jeu et pour quelles conséquences? Enfin, quels sont les facteurs susceptibles de favoriser leur émergence?

Les études classiques des politiques publiques définissent souvent l'État par la domination et le pouvoir qu'il exerce sur la société et l'économie dans un territoire (Le Galès, 1999, p. 225). Or dans les idées relatives à la gouvernance (Campbel, 2002; Calame, 1997), si celles de pilotage sont bien présentes, elles gomment le primat accordé à l'État souverain.

75 Poser la question de la gouvernance conduit donc à rechercher et à comprendre l'articulation des différents types de régulation sur un territoire, à la fois en terme d'intégration politique et sociale, et en termes de capacité d'action, ce qui revient à s'interroger sur les inter-relations entre la société civile et l'État. Ce processus de coordination d'acteurs, de groupes sociaux et d'institutions pour atteindre des buts propres qui pourraient être liés à des question de légitimité accrue renouvelle-t-il le débat autour de l'action collective et plus généralement autour des politiques publiques?

\subsection{Nature multidimensionnelle de la participation}

76 Il ne suffit pas qu'une action compte une diversité d'acteurs pour être participative. Une distinction est nécessaire entre, d'une part, les participants «passifs » qui par exemple reçoivent une information lors de débats organisés ou qui répondent à des enquêtes par questionnaires et, d'autre part, les participants « actifs ", sujets agissant du processus mis en œuvre (Baron, 2001). On a tendance à parler de « participant » sans tenir compte des différentes modalités de participation des acteurs, ce qui peut en donner une image faussée. 
77 La prise en compte du développement durable dans la gestion de la ressource en eau devrait faire évoluer la manière de gouverner en intensifiant la vie démocratique par la participation et le débat public. Le Conseil économique dans son rapport n'hésite pas à écrire qu' "une sorte de co-élaboration, de co-production des règles et des décisions peut ainsi émerger, redonnant au pouvoir politique plus de légitimité ». Il conclut même par cette phrase, «c'est de notre civilisation et de sa pérennité qu'il s'agit en définitive!» ${ }^{26}$. Cette démocratie participative articulée à la démocratie représentative est d'autant plus nécessaire que les questions environnementales sont complexes. Là encore, le CES fait le constat que « l'écoute, le dialogue ou la négociation ne semblent pas avoir été une méthode courante de gouvernement». Quels pourraient être les modalités d'une participation effective des citoyens?

78 Une vision théorique et idéaliste de la participation impliquerait que celle-ci procède de l'ensemble des acteurs du district hydrographique à toutes les phases de mise en œuvre de la DCE. En pratique, toute opération de participation à ce niveau est soumise à des limites et à des contraintes.

79 Il est évident que le choix du degré d'implication des différents acteurs d'une action publique constitue un paramètre essentiel pour mesurer le niveau de participation (Cousin et de Aert, 1992). Mais plus la participation sera large, plus l'instance organisatrice sera confrontée à la difficulté de faire travailler ensemble des acteurs aux intérêts, mais surtout, aux capacités différentes. En ce sens, la restriction de la participation aux " parties intéressées » relativise l'enjeu qui pourrait être l'amélioration de l'efficience de l'action publique ou l'instauration d'un espace de négociation ou de concertation.

80 À l'inverse, la non limitation de la participation peut tout aussi bien conduire aux mêmes effets, en raison d'éléments de blocages divers. Ceci étant dans l'absolu, trois hypothèses de participation étaient possibles :

- La première cherche la participation du plus grand nombre (largeur de la participation) à quelques étapes (profondeur de la participation) seulement de la mise en œuvre de la DCE ;

- La seconde préconise une participation à toutes les étapes de mise en œuvre de la DCE, pour un nombre restreint d'acteurs ;

- Enfin, la troisième recherche à la fois une participation large et profonde.

81 Le choix d'adopter l'une de ces hypothèses relève notamment des préférences de l'État, mais aussi des limites et contraintes engendrées par le contexte, mais définit au final l'étendue de la participation comme le montre le schéma ci-dessous. 
Figure 6. Largeur et profondeur de la participation dans le cadre de la mise en œuvre de la DCE

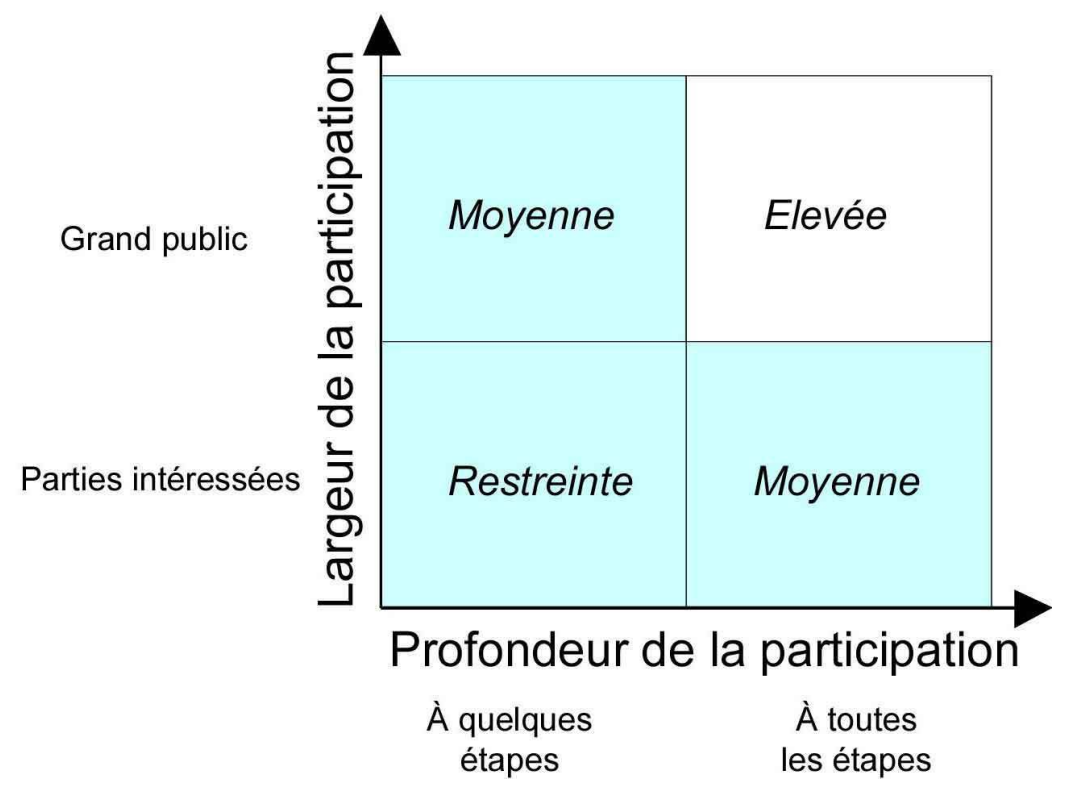

Degré de participation dans le cadre de la mise en oeuvre de la DCE

Source : Scarwell/Laganier 2003

Nous ne prétendons pas qu'il existerait un niveau idéal de participation sans prendre en compte les contingences que sont le contexte et les impératifs liés au calendrier de réalisation. Ces contraintes vont donc influer sur la largeur et la profondeur de la participation et finalement la limiter.

Figure 7. Les différents modes de participation du public

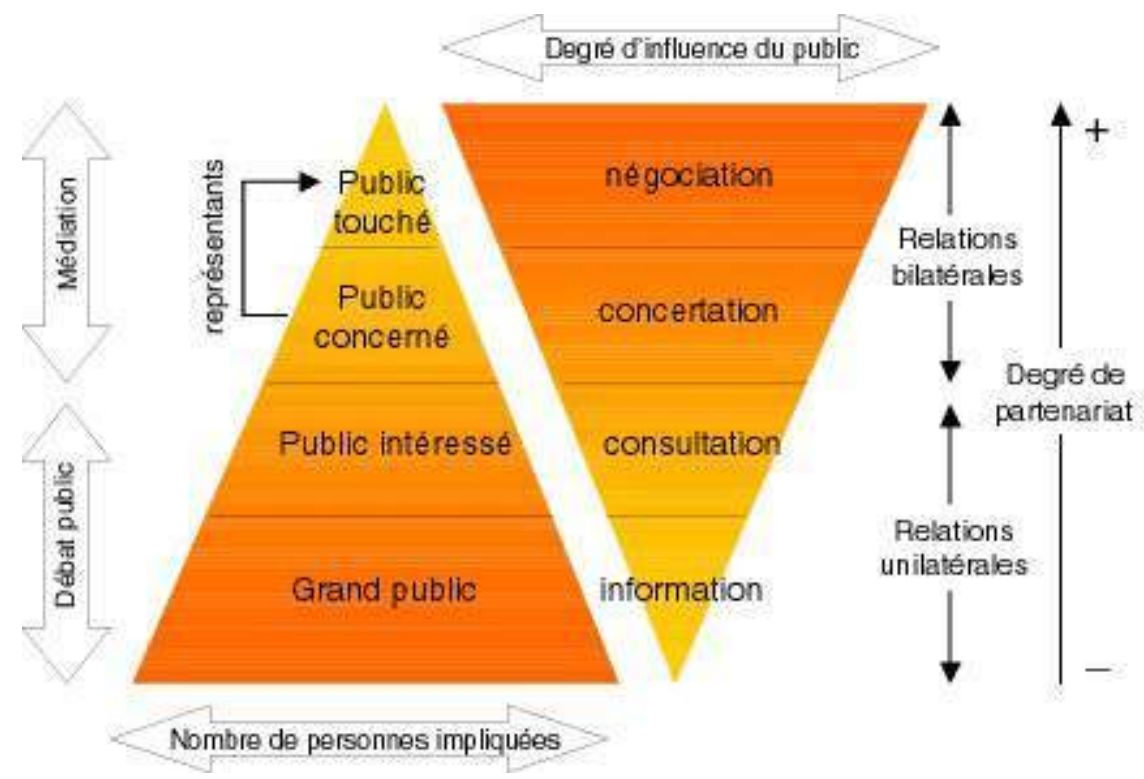

Source : Mettan N et Vodoz L. 1992) ${ }^{27}$ 
83 participation à plusieurs niveaux : relativement large pour les phases préparatoires et finales, mais plus «étroite et plus profonde pour l'élaboration des outils d'analyse et de collecte de données », comme le montre le schéma ci-dessus (figure 7).

\subsection{Des exigences de la Directive à la mise en œuvre pratique}

La mise en œuvre de l'article 14 de la DCE par l'État français se traduit concrètement par le choix d'une variation dans le degré d'implication des " groupes cibles ».

En effet, le Comité de Bassin distingue deux catégories d'acteurs: les "parties intéressées » et le «public ». De surcroît, les modalités de participation de ces deux groupes varient dans le temps et selon la nature des opérations à effectuer, comme l'attestent les figures 2 et 7.

Conformément aux exigences de la directive, les États membres doivent suivre les trois phases de la procédure de consultation du public $(2006,2007,2008)$, afin de mettre à disposition des informations et encourager la participation active des parties intéressées. Le principe de subsidiarité nationale s'applique pour les moyens à mettre en œuvre en amont, en vue d'une consultation du public effective à partir de 2006. A cet effet, la DIREN et l'Agence de l'eau Artois-Picardie ont créé une commission dans le cadre du bassin Artois-Picardie intitulée "participation du public", composée de représentants du comité de bassin, d'associations de consommateurs, de protection, de la pêche, de l'éducation à l'environnement, de l'éducation nationale, de la presse, de la commission locale de l'eau, de l'EPTB, et d'experts en communication dont le rôle est d'organiser et de favoriser la participation du public. Elle est aidée par la Commission Permanente SDAGE et milieux aquatiques, chargée de l'état des lieu ${ }^{28}$, créée préalablement.

D'autres rendez-vous d'information et de sensibilisation du public ont été organisés en dehors des instances de bassin et notamment un séminaire de "groupe lecteurs » le 7 novembre 2002, un séminaire des animateurs de SAGE le 21 novembre 2002, et enfin, un atelier "eau et relais d'opinion» le 7 décembre 2002, qui vise essentiellement les associations. À ce stade, ne sont concernées que les " parties intéressées », conformément à la dénomination empruntée à la DIREN et à l'Agence de l'eau.

À destination du grand public, plusieurs débats sont en cours dans les principales villes du bassin. Le déroulement des demi-journées est immuable et se décompose en ateliers qui se déroulent simultanément. L'un d'entre eux s'intitule «la gouvernance et les moyens » et consiste essentiellement à s'interroger sur la répartition des compétences entre les différents niveaux de collectivités et la mobilisation de celles-ci sur le périmètre des bassins versants.

Lors de ces débats publics, un questionnaire est remis aux participants et vise à " recueillir la perception sur une liste, non exhaustive, d'enjeux identifiés avec un groupe d'experts" (Strébelle, 2003) du Bassin. A l'examen de ce questionnaire, composé de cinquante et une questions, on remarquera que ne figure aucune question relative aux conditions de la participation directe du grand public concerné, de même qu'il n'est pas fait état de la différenciation entre les parties intéressées participant directement au processus du public, lequel est simplement informé plutôt que consulté.

90 Par ailleurs, les références dans la lettre d'accompagnement du questionnaire à l'enjeu collectif qu'implique la prise en charge de la gestion de l'eau par chaque individu ainsi 
qu'aux enjeux planétaires de l'eau, sont à mettre en comparaison avec les trois heures et demie de débat accordé et surtout avec l'organisation simultanée des ateliers qui ne permettent pas au grand public de s'informer davantage sur les dossiers techniques, alors même que certains documents de l'Agence de l'eau et de la DIREN insistent sur la " nécessité d'élaborer des documents non techniques ${ }^{29}$, ce qui signifie compréhensibles par tous. Il est aussi à noter qu'on demande aux interviewés, en fin de questionnaire, de classer les priorités en matière de gestion de l'eau alors qu'on s'évertue à leur présenter la DCE comme une démarche globale et intégrée.

91 Pour satisfaire à l'obligation d'information du grand public, il est prévu de s'appuyer sur les relais locaux que sont les collectivités, les organismes consulaires et les associations.

Toutes ces opérations ont pour objet de « développer une culture de la participation, un enjeu de crédibilité et de transparence » de "clarifier les règles du jeu et les limites de l'exercice », enfin, "de prendre en compte les observations du public et d'en rendre compte $»^{30}$.

Au regard des objectifs affichés par l'État que sont le développement d'une culture de la participation, d'un enjeu de transparence et de crédibilité, il convient de se demander si les modalités de la participation retenues répondent à ces enjeux.

Ce qui revient à se demander si la subsidiarité active qui est au cœur de la gouvernance est, à l'inverse, entravée par l'absence de marges de manœuvre des acteurs publics et privés et par la définition a priori des sphères publique et privée. De même, le partenariat impliquant une négociation et donc des possibilités d'initiatives de la part de chaque partenaire, il conviendra de s'interroger plus globalement sur les modalités de ce dialogue.

La création d'un espace de coopération devait conduire à assurer la transparence des décisions tout en renforçant leur légitimité. Toutefois, certaines dissymétries constituent des obstacles à un réel partenariat entre les acteurs concernés. Le fait de définir a priori des catégories d'acteurs qui se voient affecter chacun une place et un rôle dans le processus de mise en œuvre de la DCE n'est pas propre à garantir cette transparence. Comme le souligne Pierre Zémor, «les limites du public intéressé par ( un débat) ne sont pas aisément discernables. Tous les citoyens sont légitimement concernés, mais certains plus que d'autres. Il n'est pas concevable de pondérer les interventions dans des exercices de participation du public, d'autant qu'en matière d'information toute sélection s'apparente, à l'ère médiatique, à une dissimulation, à une restriction suspecte ou à une dissimulation. » (Zémor, 2003, p. 84).

Si le gouvernement s'attache par l'organisation d'un débat en trois phases à mettre en évidence un espace social visible de coopération, il n'en demeure pas moins que d'autres espaces demeurent des cercles fermés pour un grand nombre d'acteurs. La création de ces territoires concentriques de participation active les interrogations sur les lignes réelles de confrontation entre les exigences démocratiques de la participation et les nécessaires évolutions du système politico-administratif.

Telle que l'organisation se présente, l'État a délimité un territoire où seuls ceux qui sont admis pourront profiter de l'institutionnalisation de certains espaces de coopération. Alors que les pratiques de coopération et de participation sont censées lutter contre un pouvoir jugé par trop centralisé et éloigné des citoyens, les modalités du dialogue ne font que renforcer ce caractère. Il se pose alors la question de savoir si le système politique est 
à même d'évoluer en intégrant ces exigences démocratiques et en adoptant ses modalités de fonctionnement.

Sous le masque de la non coercition et de l'absence de formalisme, la participation dissimule des formes de contrôle émanant justement de cet État omniprésent. Si l'on se souvient des articulations entre les échelles d'intervention dans la mise en œuvre de la DCE en France, on constatera que l'État, par le biais du préfet et de ses services, est présent à tous les niveaux et qu'il ne favorise pas le besoin d'ouverture vers une pluralité d'acteurs $^{31}$. Comment dans ces conditions l'Union européenne peut-elle demander aux populations d'adhérer aux politiques qui leur sont prescrites ?32

Finalement, on peut se demander s'il est possible de souscrire à l'idée selon laquelle ces nouvelles formes d'action de gouvernance seraient plus efficaces ou plus efficientes que les procédés antérieurs? En effet, les voies du débat et du dialogue sont déjà définies et encadrées, et on ne voit pas comment l'État peut garantir la mise en œuvre d'un dialogue authentique et équitable entre tous les acteurs concernés. En poussant plus avant notre réflexion, on pourrait se demander si les pouvoirs publics se mobilisent de manière stratégique, avec la volonté de mettre en œuvre de nouvelles politiques, ou s'ils ont seulement le souhait de faire évoluer leur discours pour être «dans l'air du temps » et capter de nouvelles opportunité ou également, si le développement des procédures d'information et de concertation ne vise pas à réduire des menaces de contestation (Habermas, 1997) ? Le cadre procédural de la gouvernance maintient ici une démarche essentiellement « top-down ».

Enfin, la durabilité affichée à travers les textes législatifs coïncide-t-elle avec une vision élargie et pertinente pour répondre aux interrogations majeures des citoyens. En effet, sous l'influence de la décentralisation émergent des politiques publiques locales qui, pour gérer au mieux leurs attentes, au sein d'une société de plus en plus complexe, utilisent la notion de gouvernance et de nombreux principes de la durabilité.

\section{Conclusion}

101 L'observations en temps réel de la mise en œuvre de la DCE et des débats qui ont été organisés dans ce cadre ont permis de soulever des interrogations sur le traitement de la participation du public par l'État. On retiendra que l'approche ambiguë qu'à celui-ci de la participation laisse en dehors du débat démocratique les exigences même de la participation. La logique de participation doit permettre aux populations de peser réellement sur le débat et de se mobiliser comme allié, par le développement de toute une gamme de dispositifs d'information, de communication d'éducation, de participation.

102 En effet, si de nombreux articles et analyses font état depuis plusieurs années de l'intérêt des Français pour les questions environnementales (Bourg, 1993 ; IFEN, 2002), on fera le constat que ce nouveau degré d'exigence des citoyens n'est pas toujours pris en compte. Or, si l'État semble tenir compte des impératifs économiques dans l'élaboration des décisions publiques concernant l'environnement, il demeure encore réticent à en élargir le champ des acteurs.

103 La mise en visibilité d'espaces de coopération ou de participation du public ne saurait justifier l'existence d'une nouvelle régulation de l'action publique ou n'être prise en compte que pour la part de légitimité qu'ils peuvent redonner à l'intervention publique, alors que "plus on remonte en amont, plus les processus décisionnels paraissent fermés» 
(Rumpala, 2003, p. 365). En l'occurrence, l'apprentissage institutionnel est encore marqué par la prégnance de schémas "bureaucratiques» ou des logiques d'actions qui en découlent. Pourtant, comme le souligne Yannick Rumpala, «l'évolution vers un État procédural» (Rumpala, 2003, p. 366) et la montée des logiques de gouvernance (Leca, 1996), le brouillage des frontières entre privé et public (Le Galès, 1995) invitent l'État à reconsidérer les modalités d'élaboration de l'action publique et ne peuvent simplement relégitimer ses interventions en laissant percevoir de nouveaux espaces de discussion.

Finalement, le décloisonnement relatif des niveaux territoriaux et des réseaux d'acteurs qui structurent les décisions ne nous a pas permis de percevoir un renouvellement dans les modalités de la coopération. Certes, de nouvelles articulations entre territoires signalent un traitement plus territorial de la gestion de l'Escaut, mais elles n'engendrent pas de changement d'équilibre entre les acteurs territoriaux dans les modalités de régulation des problèmes.

\section{BIBLIOGRAPHIE}

Assouline G., Blancher Ph. (2003), Démocratie délibérative et décisions publiques en Europe : le cas des déplacements urbains, Dossier 2001 Plus, Centre de Prospective et de Veille Scientifique, DRAST, ministère de l'Équipement, 60 pages, à paraître.

Badie B. (1995), La fin des territoires, Essai sur le désordre international et sur l'utilité sociale du respect, Paris, Fayard,

Baron G., (2001), Évaluation, participation, apprentissage dans l'action publique, Paris, L'Harmattan.

Bourg D., (1993), (dir), La nature en politique ou l'enjeu philosophique de l'écologie, Paris, L'Harmattan.

Calame P. et Talman A. (1997), L'État au cour, Desclée de Brower.

Calame P., (2003), La démocratie en miettes, éditions de la Fondation Charles Léopold Mayer.

Campbel B., (2002), « Gouvernance, réformes institutionnelles et redéfinition du rôle de l'État : quelques enjeux conceptuels et politiques soulevés par le projet de gouvernance décentralisée par la Banque mondiale », in « Principe de gouvernance » cahier de propositions de l'Alliance, éditions Charles Léopold Mayer.

Cousin B., et de Eart L., (1992), The case for participatory evaluation, educational evaluation and policy analysis, vol. .14, $\mathrm{n}^{\circ} 4$, pp. 397-418.

Crozier M. et Friedberg E., (1977), L'acteur et le système, Paris, édition du Seuil, collection point Points Essais.

de la Malène C., (1997) L'application du principe de subsidiarité, rapport nº 46 du Sénat.

Freyss J, (2003), Décentralisation et mondialisation, Cahiers du Gemdev, à paraître.

Gaudin J.-P., (1996), (dir), La négociation des politiques contractuelles, Paris, L'Harmattan.

Godard O. (1994), «Le développement durable : paysage intellectuel », in Natures - Sciences -

Sociétés, 2, (4), p. 309-322. 
Habermas J,(1997), Théorie de l'agir communicationnel, Paris, Fayard.

IFEN (2002), « Les attentes des Français en matière d'environnement », (consulter la page http:// www.ifen.fr/pages/4ecosoc.htm)

Jacquet P., (2000), « La construction européenne entre mondialisation et instances nationales », in Taillard M., Thérer B. et Uri D., (dir.), Innovations institutionnelles et territoires, Paris L'harmattan.

Jouve B. (1994)., Urbanisme et frontière. Le cas franco-genevois, Paris, L'Harmattan,

Le Galès P., (1995), «Introduction : les réseaux d'action publique entre outil passe-partout et théorie de moyenne portée » in Le Galès P. et Thatcher M. (dir), Les réseaux de politique publique. Débat autour des policy networks, Paris, L'Harmattan.

Le Galès P. (1995), « Du gouvernement des villes à la gouvernance urbaine », revue française de Science Politique, $\mathrm{n}^{\circ} 45,1$, pp. 57-95

Le Galès P. (1999), « Régulation, gouvernance et territoire », in Jobert B. et Commaill J. (dir.), Les métamorphoses de la régulation politique, LGDJ.

Leca J., (1996), « La 'gouvernance' de la France sous la V ${ }^{\mathrm{e}}$ République : une perspective de sociologie comparative ", in d'Arcy F. Rouban L. (dir), De la V République à l'Europe. Hommage à JeanLouis Quermonne, Paris, Presses de la FNSP.

Lefèvre. C (1995) , «Le gouvernement des aires métropolitaines dans les pays industrialisés », 2000 Plus, DRAST, Ministère de l'Équipement, n 33 , pp. 3-24

Leresche J.P, Levy R. (dir.) (1995), La Suisse et la coopération transfrontalière, repli ou redéploiement ?, Zurich, ed. Seismo,

Levrat N. (1994), Le droit applicable aux accords de coopération transfrontalières entre collectivités publiques infra-étatiques, Paris, PUF.

Loriferme M. (1987), 40 ans de politique de l'eau, Paris Economica,

Noël C. (2003), « Que prévoit la Directive Cadre sur l'Eau en matière de participation du public ?», (http://www.eau-artois-picardie.fr/europedeleau/dce/relaisdopinion/index.htm)

Pongy M, (1997), « Gouvernance et citoyenneté, la différenciation du politique », in Saez G. Leresche J.-P. et Bassand M. (dir) Gouvernance Métropolitaine et transfrontalière : action publique territoriale, logiques politiques, Paris, L'harmattan.

Raffestin C. (1993), « Autour de la fonction sociale de la frontière », Espaces et Sociétés, n 70-71, pp. 157-164

Rapport du CES, (2002), « Environnement et développement durable : l'indispensable mobilisation des acteurs économiques et sociaux », décembre 2002.

Robert P, (1995), «Les normes de l'État-Nation, une hégémonie enviée », in Robert P. ;. Sach F, Normes et déviances en Europe, Un débat Est-Ouest, Paris, L'Harmattan.

Rumpala Y., (2003), Régulation publique et environnement, questions écologiques et réponses économiques, Paris, L'Harmattan.

Saez G ; Bassand M., (1997), « Les recompositions de l'action publique en contexte métropolitain et transfrontalier ", in Saez G. ; Bassand M. et Leresche J.-P., Gouvernance métropolitaine et transfrontalière, action publique territoriale, Paris, L'Harmattan.

Stoker G., (1998), « Cinq propositions pour une théorie de la gouvernance », in Revue internationale des sciences sociales, $\mathrm{n}^{\circ}$ 155, mars 1998. 
Strébelle A.,(2003), Lettre « débats sur l'eau », directeur de l'Agence de l'Eau, Artois- Picardie.

Theys J., (2002), « La gouvernance, entre innovation et impuissance : le cas de l'environnement », in Wachter S. L'aménagement durable : défis et politiques, éditions de l'Aube, pp. 125 -167.

Valiron F. (1990), La politique de l'eau en France de 1945 à nos jours, Presses de l'ENPC.

Zémor P., (2003), Pour un meilleur débat public, Paris, Presses de Sciences Po.

\section{ANNEXES}

Liste des sigles :

DCE : Directive Cadre sur l'Eau

SDAGE : Schéma Directeur d'Aménagement et de Gestion de l'Eau

SAGE : Schéma d'Aménagement et de Gestion de l'Eau

DE : Direction de l'Eau

DIREN : Direction Régionale de l'Environnement

CLE : Commission Locale de l'Eau

CIE : Commission Internationale de l'Escaut

CIPE : Commission Internationale pour la Protection de l'Escaut

COPIT : Conférence Permanente Intercommunale Transfrontalière

TSC :Technical Schelde Commission

EPTB : Etablissement Public Territorial de Bassin

\section{NOTES}

1. JOCE L.327/1 du 22 décembre 2000.

2. Loi sur l'eau du 16 décembre 1964.

3. Loi $n^{\circ}$ 92-3 du 3 janvier 1992, modifiée par la loi n 92-1336 du 16 décembre 1992 et par la loi $n$ - 95-101 du 21 février 1995 - JO des 4 janvier 1992, 23 décembre 1992 et 3 février 1995.

4. SDAGE : Schéma Directeur d'Aménagement et de Gestion de l'Eau définissant une planification stratégique à l'échelle de la circonscription de bassin et après application de la DCE, à l'échelle du district hydrographique.

5. SAGE : Schéma d'Aménagement et de Gestion de l'Eau définissant une planification opérationnelle à l'échelle du bassin versant.

6. Selon Coralie Noel, ingénieur chargé de mission à la Direction de l'eau, cette participation active s'appuiera avant tout sur les structures de concertations existantes : Comité de Bassin, Commission locale de l'eau ... composée des représentants des collectivités, de l'État et des usagers (associations, industriels, agriculture etc..)

7. Cf. Convention d'Aarhus du 23 juin 1998 sur l'accès à l'information, la participation du public au processus décisionnel et à l'accès à la justice en matière d'environnement. Elle fut transcrite dans le droit français par la loi n² 2002-285 du 28 février 2002 dite loi « démocratie et proximité » autorisant l'approbation de la Convention sur l'accès à l'information, la participation du public au processus décisionnel et à l'accès à la justice en matière d'environnement. 
8. Article 3- 1- a de l'Accord international sur l'Escaut de Gand du 3/12/2002.

9. Notamment l'article 13 et l'article 6.

10. Accord international sur l'Escaut, Gand, 3/12/ 2002.

11. Cf. Accord international sur l'Escaut du 3 décembre 2002 signé à Gand.

12. Premier accord de coopération transfrontalière sur l'Escaut instituant la CIPE (commission internationale de protection de l'Escaut) : accord de Charleville-Mézières.

13. La délégation française est composée de la Diren, la DDE, VNF, la DRIRE (statut d'observateur), l'Agence de l'eau Artois-Picardie, les communautés urbaines de Dunkerque et de Lille.

14. Il s'agit des Communautés urbaines de Dunkerque et de Lille.

15. Art.2 (b) de l'accord international de Gand.

16. L'union européenne présente la gouvernance comme «les règles, les procédures et les comportements qui influent sur l'exercice des pouvoirs au niveau européen, particulièrement du point de vue de l'ouverture, de la participation, de la responsabilité, de l'efficacité et de la cohérence ", in Com 428, Bruxelles le 25/07/2001, « Livre blanc sur la gouvernance européenne », 2000.

17. Livre blanc sur la gouvernance, chantier $n^{\circ} 1$ : «Accroître la qualité du débat public européen ", mai 2001 p. 1 ; Com 428, Bruxelles le 25/07/2001, «Livre blanc sur la gouvernance européenne», 2000, mais aussi cf. Hergenhan J.(2001), «Quelle gouvernance pour l'Union européenne après Nice? » in Eurocities magazine, $\mathrm{n}^{\circ} 13$.

18. Dans le traité de Maastricht de 1992, la notion de subsidiarité est définie en modification du Traité de Rome (art.3B) «dans les domaines qui ne relèvent pas de sa compétence exclusive, la Communauté n'intervient, conformément au principe de subsidiarité, que si et dans la mesure où les objectifs de l'action envisagée ne peuvent pas être réalisés de manière suffisante par les États membres et peuvent donc, en raison des dimensions ou des effets de l'action envisagée, être mieux réalisés au niveau communautaire $»$.

19. Selon P. Calame, la subsidiarité active «n'est pas un simple enrichissement du principe de subsidiarité. Elle partage avec lui le souci de respecter et valoriser la diversité, donc les approches locales, mais elle en diffère radicalement dans le traitement des interdépendances ", ou encore « a chaque échelle de la gouvernance, la subsidiarité active ne consiste pas à appliquer des règles uniformes ni, à l'inverse, à agir en toute liberté mais à trouver des solutions à des objectifs communs et selon des principes définis de manière collective, op. cit., p. 182 et 192

20. COPIT : Conférence permanente intercommunale transfrontalier créée en 1991 pour favoriser la concertation et la coopération entre les intercommunales belges de Tournai, Courtrai, YpresRoulers, Mouscron et Lille-Métropole Communauté urbaine ; TSC/Technical schelde Commission - commission technique de l'Escaut pour la partie aval du bassin de l'Escaut.

21. Le préambule 14 de la DCE énonce «le succès de la présente directive requiert l'information, la consultation et la participation du public»

22. Circulaire du 12 février 2002 qui précise qu'il est nécessaire d'identifier les attentes du public et de préparer le terrain par une sensibilisation appropriée.

23. Cf. le site du ministère de l'écologie et du développement durable.

24. Deux observations viennent à l'esprit d'une part l'administration d'État est elle-même en proie à la différenciation et cherche à s'autonomiser au niveau régional, d'autre part, les institutions étatiques régionalisés sont et seront peut-être de plus en plus concurrencées par une série d'organisme ad hoc et d'acteurs qui pourraient faire basculer ce modèle fragilisé.

25. Si l'on suit Jessop B. (1995) «la gouvernance renverrait aux idées de conduite, de pilotage, de direction. Sur ce point voir aussi Sennellart M., (1995, Les arts de gouverner », Le Seuil, Paris.

26. Cf. Rapport du CES, (2002), «Environnement et développement durable : l'indispensable mobilisation des acteurs économiques et sociaux », décembre 2002. 
27. La place de la négociation dans le processus d'aménagement du territoire et de protection de l'environnement, in La négociation : son rôle, sa place dans l' d'aménagement du territoire et de protection de l'environnement, sous la direction de Ruegg J., pp. 79-94. Cette figure est reprise par Dziedzicki J.M.): Médiation environnementale: des expériences internationales aux perspectives dans le contexte français, Actes du séminaire «Concertation, décision et environnement » Paris, pp. 35-60. C'est cette reprise que nous avons insérée dans notre texte.

28. Cf. Discours du 7 décembre 2001 du Préfet de bassin.

29. Cf. document commun de l'Agence de l'eau et de la DIREN, 21 novembre 2002 de Déricq C., et de Meerpoel S.

30. Cf. document commun de l'Agence de l'eau et de la DIREN, 21 novembre 2002 de Déricq C. et de Meerpoel S.

31. Pour un schéma théorique plus général, voir Stoker G., (1998), Cinq propositions pour une théorie de la gouvernance, Revue internationale des sciences sociales $n^{\circ} 155$, mars.

32. Voir le $n^{\circ} 24$ des Dossiers techniques, Territoires et Société, intitulé «la production de l'assentiment dans les politiques publiques", ministère de l'Équipement/DRAST/. Centre de Prospective et de Veille Scientifique, 1993.

\section{RÉSUMÉS}

La notion de développement durable semble bénéficier depuis quelques années d'un contexte favorable à sa mise en œuvre tant au niveau local qu'aux niveaux communautaire et étatique. L'observation et l'analyse d'un certain nombre de politiques locales élaborées dans un tel contexte posent toutefois la question de leur pertinence au regard des enjeux du développement durable et de leur capacité réelle à faire évoluer en profondeur l'action publique locale. En d'autres termes, elles soulèvent le problème de leurs impacts effectifs. L'objectif de cette contribution est d'analyser comment dans un espace densément peuplé, le district international de l'Escaut, la question de la gestion de l'eau concerne le développement durable, en lui imposant des cadres territoriaux complexes et mouvants, et comment la mise en œuvre des principes de développement durable se construit sur l'émergence du souci de proximité et influence les politiques de l'eau.

The concept of sustainable development appears to be enjoying a favorable context in terms of implementation at local government level as well as European and national levels. The study of local policies in this context begs the question of their efficiency in relation to the stakes of sustainable development as well as their capacity to change in depth local policies. The aim of this paper is to analyze how it is possible in a densely populated area, i.e. the river basin district of Escaut, to demonstrate that water management is connected with sustainable development within changing local frameworks. We will also examine how the principles of sustainable development rely on proximity and determine water policy. 


\section{INDEX}

Keywords : reorganization of space, Governance, Proximity, border, framework directive with field of water policy, river basin district, international commission of Escaut

Mots-clés : recomposition des territoires, directive-cadre sur l'eau, commission internationale de l'Escaut, district hydrographique, gouvernance, proximité, frontière

\section{AUTEURS}

\section{HELGA-JANE SCARWELL}

Helga-Jane Scarwell, maître de conférences en Sciences politiques à l'Université de Lille 1, spécialisée dans le domaine des politiques publiques environnementales. Laboratoire de Géographie des Milieux Anthropisés - UMR 8141 CNRS

\section{RICHARD LAGANIER}

Richard Laganier, professeur de géographie à l'Université de Paris 7 et secrétaire de la commission « Hydrosystèmes continentaux » du Comité national français de géographie, est spécialisé dans le domaine de la gestion de l'eau. Il vient de soutenir une habilitation à diriger des recherches sur le thème " l'interface eau et territoire dans le nord de la France ». Laboratoire de Géographie des Milieux Anthropisés - UMR 8141 CNRS 